\title{
A case of nodal marginal zone B-cell lymphoma of the lower eyelid
}

\author{
Cho Long Lee, \\ Il Seok, Lee, \\ Sung Gyun Jung \\ Department of Plastic and \\ Reconstructive Surgery, Eulji University \\ College of Medicine, Daejeon, Korea
}

\begin{abstract}
A marginal zone B-cell lymphoma is a type of B-cell lymphoma which is normally located at the margins of the secondary lymph node follicles. According to 2008 World Health Organization (WHO) classification, there are three main types classified according to the location of invasion: nodal lymphoma in the lymph nodes, splenic lymphoma in the spleen, and extranodal lymphoma in other locations. Recently, we have experienced a rare case of primary nodal marginal zone lymphoma that arose in the lower eyelid. Therefore, we report this case with a review of literature.
\end{abstract}

Keywords: B-cell / Lower eyelid / Lymphoma

\section{INTRODUCTION}

Marginal zone B-cell lymphoma (MZL) is a type of lymphoma normally located at the margins of the secondary lymph node follicles. The most common subtype is extranodal, accounting for $50 \%$ to $70 \%$ of MZLs. According to the World Health Organization (WHO) classification, MZL is divided into three types according to the primary site: nodal, extranodal, and splenic. The splenic and the nodal types have been reported to account for $20 \%$ and $1 \%$ of MZLs, respectively [1]. The authors report a case involving a man with a mass on his left lower eyelid, who underwent excision and biopsy, and was diagnosed with marginal B-cell lymphoma in a lymph node.

\section{CASE REPORT}

A 77-year-old man was presented to our hospital with a mass on the left lower eyelid that developed gradually over a 2-year period. The patient had no complaints of any ophthalmic symptoms such as eye dryness, or B symptoms of weight loss,

\section{Correspondence: Sung Gyun Jung}

Department of Plastic and Reconstructive Surgery, Eulji University College of

Medicine, 95 Dunsanseo-ro, Seo-gu, Daejeon 35233, Korea

E-mail: psjung111@gmail.com

Received June 2, 2020 / Revised July 25, 2020 / Accepted August 18, 2020 fever, or night sweats. He did, however, have medical histories of hypertension ( 5 years previously), benign prostatic hyperplasia (3 years previously), and smoking (20 pack-years). There was no family history.

Physical examination revealed a solid, fixed, and painless mass, measuring approximately $2.5 \times 1.5 \mathrm{~cm}$ in size, on the left lower eyelid (Fig. 1). Abnormal lymph nodes were not palpable in the peripheral neck. Preoperative facial ultrasonography revealed a low echoic mass, measuring $2.3 \times 1.4 \times 1.8 \mathrm{~cm}$ in size, with a relatively clear border and increased vascularity (Fig. 2). Excision and biopsy were performed for accurate diagnosis and treatment (Fig. 3). At the time of surgery, the mass penetrated the orbital septum to the orbital fat. However, no involvement of the orbit and orbital appendage was apparent. Despite the huge size of the mass, the wound was able to be reconstructed with primary closure.

Histopathologically, hematoxylin and eosin (H\&E) staining clearly differentiated the capsule (Fig. 4). Neoplastic cells exhibited irregularly shaped nuclei and a moderately abundant pale cytoplasm. Neoplastic marginal zone cells infiltrated a reactive germinal center and disrupted the follicle.

Immunohistochemical staining revealed CD20 and BCL2 positivity in the tumor cells. Tumor cells were negative and the reactive germinal center exhibited positivity for CD10 staining. 


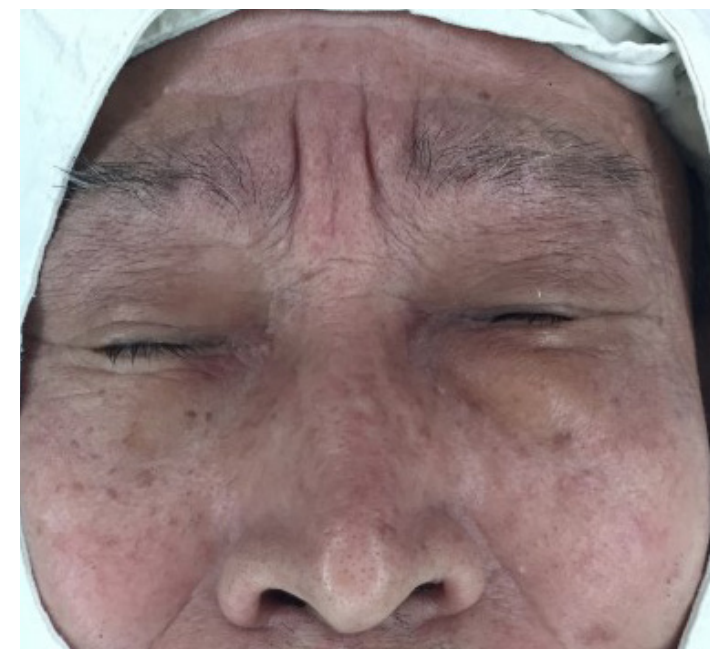

Fig. 1. Preoperative image. A 77-year-old man with a solitary mass on left lower eyelid.

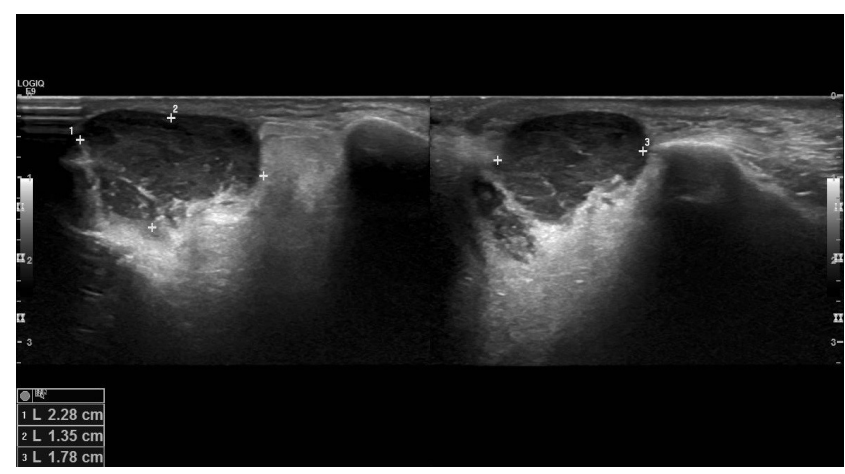

Fig. 2. Ultrasonography of the tumor.

On Ki-67 staining, the tumor cells exhibited a low proliferation index consistent with MZL (Fig. 5).

The patient was transferred to the Department of Hematology and Oncology for further clinical staging. Studies were carried out with computed tomography (CT) of skull, neck, chest and abdomen revealing lower conjunctival thickening and focal soft tissue thickening. Once identified the nature of the disease, stage 1 (Ann Arbor System), and considering the location of the lesion, 18 cycles of radiotherapy was planned.

After the 18 cycles of radiotherapy, a follow-up CT showed regression of the lower conjunctival thickening. Four months after surgery, there was no local recurrence or distant metastasis. Despite the huge loss of volume, there were no complications of disfiguration such as ectropion or facial palsy.

\section{DISCUSSION}

Nodal MZL is the rarest of the three subtypes of MZLs. In terms of morphological and immune phenotype, there is no

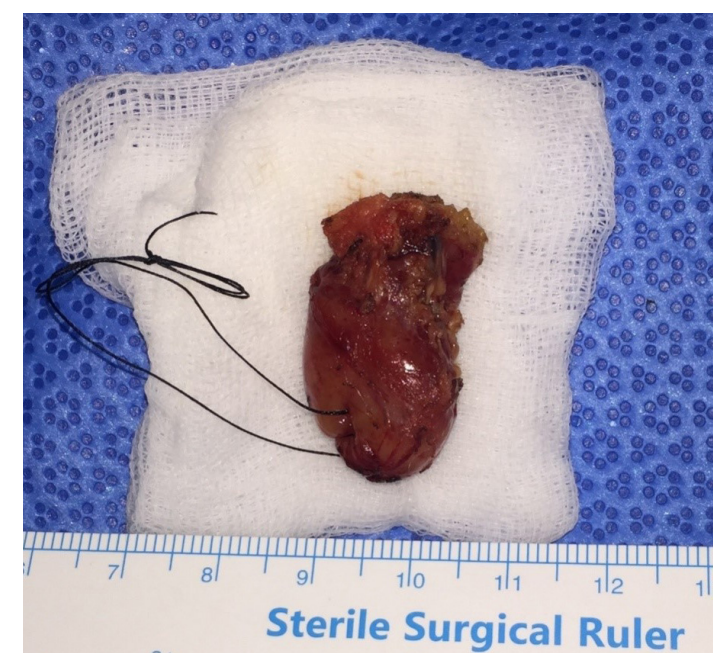

Fig. 3. Intraoperative image of the tumor.

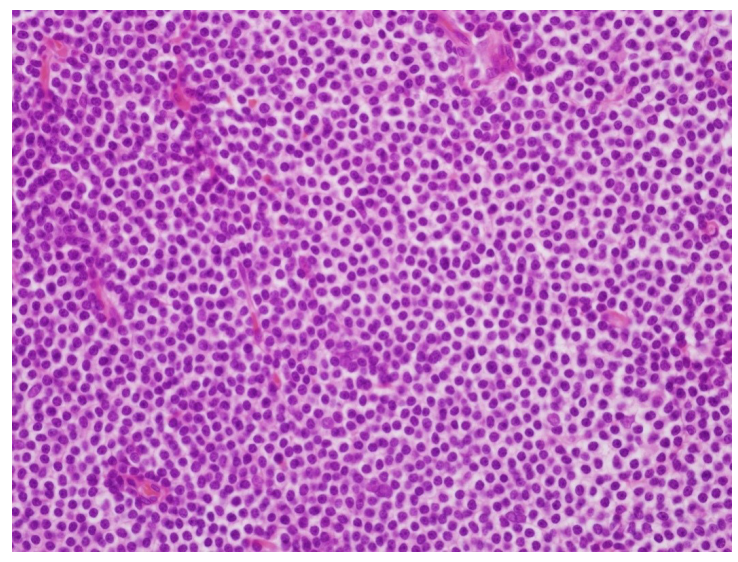

Fig. 4. Histopathology of the specimen. The neoplastic cells have irregularly shaped nuclei and moderately abundant pale cytoplasm. Neoplastic marginal zone cells infiltrate around a reactive germinal center and disrupt the follicle $(\mathrm{H} \& \mathrm{E}, \times 400)$.

difference from other subtypes, and it is classified according to the primary site.

MZLs are caused by accumulation of lymphoid tissues in organs with chronic antigenic stimulation by autoantibodies or microbial pathogens [1]. It is also associated with autoimmune diseases. Hepatitis $\mathrm{C}$ virus infection is known to contribute to the development of MZLs of all subtypes [2-6].

This patient was admitted to our clinic due to a mass in the left lower eyelid, which was diagnosed as hypervascular tumor or an inflammatory lesion using preoperative facial ultrasonography, followed by excision and biopsy. However, postoperative pathological examination, including immunohistochemistry, revealed an MZL. Additional follow-up examinations revealed no suspicious primary site. Pathological examination revealed that the mass was clearly differentiated from the surrounding 

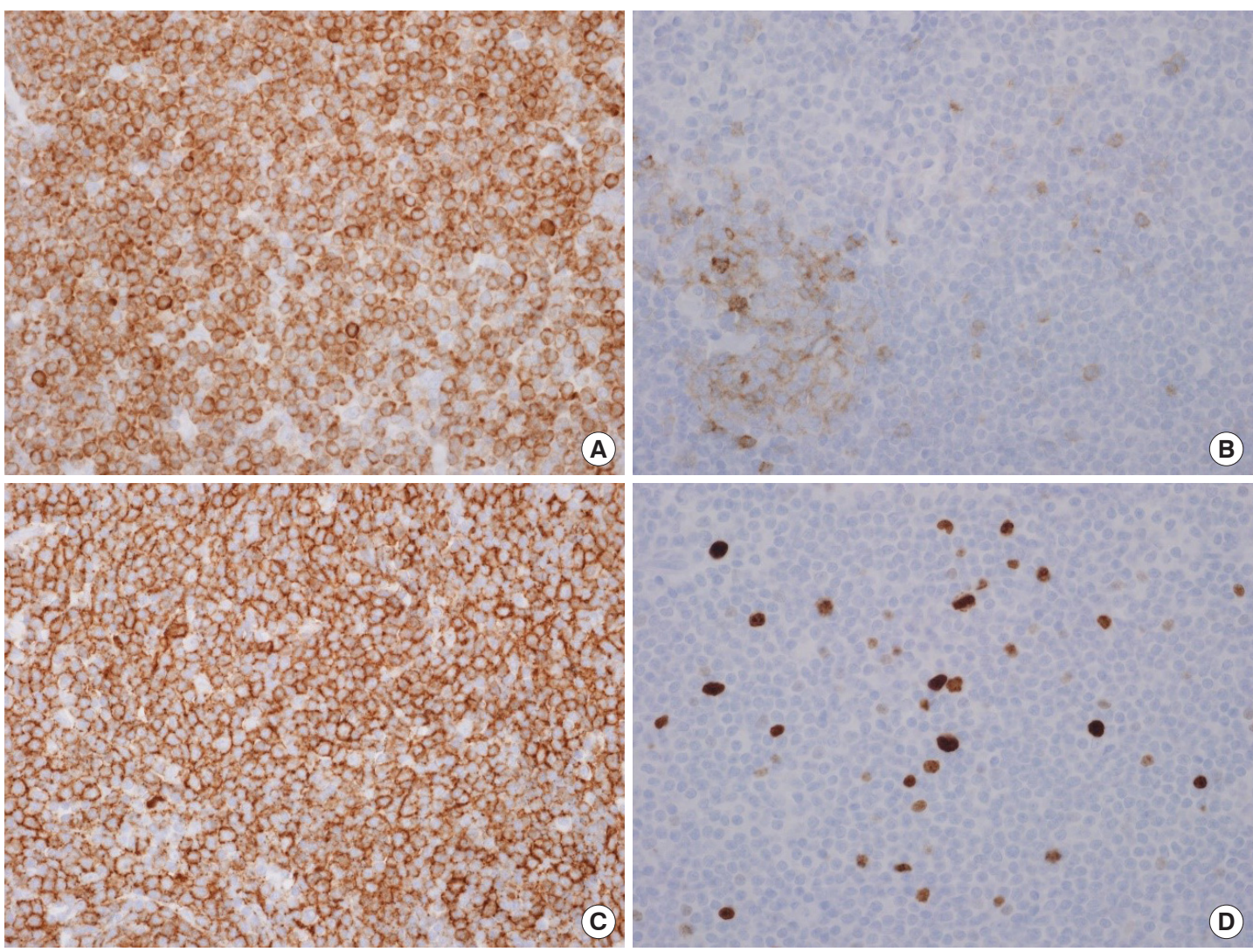

Fig. 5. Immunohistochemistry of the specimen. The tumor cells are positive in BCL2 stain (A) and CD20 stain (C) ( $\times 400)$. The tumor cells are negative, whereas the reactive germinal center is positive in the CD10 stain (B). The tumor cells show low proliferation index in the Ki-67 stain (D) $(\times 400)$.

tissue through the capsule, and that neoplastic marginal zone cells infiltrated a reactive germinal center and disrupted the follicle. Nodal MZL of the infraorbital lymph node was diagnosed.

Nodal MZL is morphologically similar to splenic or extranodal lymphoma but can be diagnosed if there is no evidence of lymph node or splenic involvement. Most cases of lymphoma are accompanied by enlargement of multiple cervical lymph nodes, which may be helpful in diagnosis. However, it is easy to overlook the possibility of lymphoma in cases involving a submandibular mass.

The most common clinical manifestations of MZL are $\geq 1$ lymphadenopathies, most of which are painless. In some cases, it may be accompanied by general weakness and B symptoms; however, there are no symptoms specific to nodal MZL.

The incidence of MZL is highest in the age range of 50 to 62 years, with a slightly higher incidence in women and marrow involvement in approximately $44 \%$. Diagnosis is determined by excluding other subtypes of MZL according to the primary site of the tumor, H\&E staining, and immunochemical cytological findings [1]. The most common site is the cervical lymph node, followed by the inguinal, thoracic, and abdominal lymph nodes. Approximately $24 \%$ of MZLs are associated with hepati- tis $C$ virus infection [7].

In the present case, a painless mass in the left lower eyelid area was the only symptom, and there was no hypertrophy of surrounding neck lymph nodes, nor was there general weakness, B symptoms, or mucosal dryness. Hepatitis $\mathrm{C}$ virus antibody was negative in serological tests.

Immunohistochemical staining revealed CD20 and Bcl-2 positivity and, because $\mathrm{CD} 10$ staining was negative, the mass could be diagnosed as nodal MZL.

Due to the low incidence of N-type MZL, there is no definitive treatment; however, multidrug chemotherapy, including anthracycline, has been proposed based on rituximab [8] Moreover, localized lesions have been reported to respond well to localized radiotherapy [9].

The complete remission rate through primary treatment is approximately $50 \%$, similar to the splenic subtype, but lower than the extranodal subtype. The 5-year survival rate is reported to be $55 \%$ to $75 \%[1,7]$.

Although prognostic factors are not well established, there have been reports that prognosis is related to the systemic status of the patient and treatment response to primary treatment [7]. The patient described in the present report underwent chemo- 
therapy with radiotherapy after surgery, with no evidence of local recurrence or distant metastasis.

We report a case of nodal MZL of the lower eyelid, which is not a multiple lymph node invasion. To our knowledge, no similar cases have been published in the literature. As such, this case report may serve as a reference for plastic and reconstructive surgeons who diagnose and treat skin tumors of the head and neck.

\section{NOTES}

\section{Conflict of interest}

No potential conflict of interest relevant to this article was reported.

\section{Ethical approval}

The study was approved by the Institutional Review Board of Samsung Medical Center (IRB No. 2020-01-001-002) and performed in accordance with the principles of the Declaration of Helsinki. Written informed consent was obtained.

\section{Patient consent}

The patient provided written informed consent for the publication and the use of his images.

\section{ORCID}

Cho Long Lee https://orcid.org/0000-0002-6441-0514

Il Seok Lee Sung Gyun Jung https://orcid.org/0000-0002-8313-2513

https://orcid.org/0000-0002-7768-9972

\section{REFERENCES}

1. Thieblemont C. Clinical presentation and management of marginal zone lymphomas. Hematology Am Soc Hematol Educ Program 2005;2005:307-13.

2. Ferreri AJ, Guidoboni M, Ponzoni M, De Conciliis C, Dell'Oro S, Fleischhauer K, et al. Evidence for an association between Chlamydia psittaci and ocular adnexal lymphomas. J Natl Cancer Inst 2004;96:586-94.

3. Cerroni L, Zochling N, Putz B, Kerl H. Infection by Borrelia burgdorferi and cutaneous B-cell lymphoma. J Cutan Pathol 1997;24:457-61.

4. Lecuit M, Suarez F, Lortholary O. Immunoproliferative small intestinal disease associated with Campylobacter jejuni. Med Sci (Paris) 2004;20:638-40.

5. Arcaini L, Paulli M, Boveri E, Vallisa D, Bernuzzi P, Orlandi E, et al. Splenic and nodal marginal zone lymphomas are indolent disorders at high hepatitis $\mathrm{C}$ virus seroprevalence with distinct presenting features but similar morphologic and phenotypic profiles. Cancer 2004;100:107-15.

6. Farinha P, Gascoyne RD. Helicobacter pylori and MALT lymphoma. Gastroenterology 2005;128:1579-605.

7. Traverse-Glehen A, Bertoni F, Thieblemont C, Zucca E, Coiffier B, Berger F, et al. Nodal marginal zone B-cell lymphoma: a diagnostic and therapeutic dilemma. Oncology (Williston Park) 2012;26:92-104.

8. Koh LP, Lim LC, Thng CH. Retreatment with chimeric CD 20 monoclonal antibody in a patient with nodal marginal zone Bcell lymphoma. Med Oncol 2000;17:225-8.

9. Berger F, Felman P, Thieblemont C, Pradier T, Baseggio L, Bryon PA, et al. Non-MALT marginal zone B-cell lymphomas: a description of clinical presentation and outcome in 124 patients. Blood 2000;95:1950-6. 